\title{
Photonic Crystal Fiber Sensors for Strain and Temperature Measurement
}

\author{
Jian Ju and Wei Jin \\ Department of Electrical Engineering, The Hong Kong Polytechnic University, Hung Hom, Kowloon, Hong Kong \\ Correspondence should be addressed to Wei Jin, eewjin@polyu.edu.hk
}

Received 27 February 2009; Accepted 27 May 2009

Recommended by Christos Riziotis

\begin{abstract}
This paper discusses the applications of photonic crystal fibers (PCFs) for strain and temperature measurement. Long-period grating sensors and in-fiber modal interferometric sensors are described and compared with their conventional single-mode counterparts. The strain sensitivities of the air-silica PCF sensors are comparable or higher than those implemented in conventional single-mode fibers but the temperature sensitivities of the PCF sensors are much lower.
\end{abstract}

Copyright ( $\odot 2009$ J. Ju and W. Jin. This is an open access article distributed under the Creative Commons Attribution License, which permits unrestricted use, distribution, and reproduction in any medium, provided the original work is properly cited.

\section{Introduction}

Recently, there has been great interest in the development of photonic crystal fibers (PCFs) and related devices and sensors. PCFs are typically made of single silica material and comprised of a solid or hollow core surrounded by a periodic array of air holes running along their length [1]. PCFs may be regarded as falling into two broad categories: the index-guiding PCFs (IG-PCFs) and the photonic bandgap fibers (PBFs). In IG-PCFs, the effective refractive index of the microstructured cladding is reduced by the introduction of the air columns to below the index of the central core, and this makes it possible to guide light in the core by a modified form of total internal reflection. Figures 1(a) and 1(b) show two examples of such IG-PCFs. They are endlessly single-mode PCFs (Figure 1(a)) [2] that support only the fundamental mode over the entire wavelength range, and highly birefringent (Hi-Bi) PCFs (Figure 1(b)) [3] that have a high birefringence and a low temperature dependence of the birefringence. In PBFs, light is confined to a lower index core by reflection from the photonic crystal cladding, which possesses out of plane photonic bandgaps and does not allow light signals with certain wavelengths and propagation constants to escape from the central core. Figures 1(c) and 1(d) show, respectively, an air-silica PBF with an air core [4] and an all solid PBF with a silica core and a cladding comprising of an array of higher index rods (Ge-doped silica) in a silica matrix [5].

The emergence of PCFs opens the door for new possibilities in ultra-broadband transmission, high-power optical fiber amplifiers and lasers, optical fiber sensors, and so forth. PCF sensors in various forms have been demonstrated for the measurement of strain [7], temperature [8], refractive index [9], bending [10], and gas concentration [11]. In this paper, we describe mainly two classes of PCF-based sensors, that is, in-fiber long-period grating (LPG) sensors and in-fiber modal interferometric sensors; we discuss their applications for strain and temperature measurement.

\section{Long-Period Grating Sensors}

An LPG is formed by periodical perturbing the refractive index or waveguide geometry longitudinally along the length of an optical fiber, with a typical period from several hundreds micrometers to $1 \mathrm{~mm}$. An LPG couples light resonantly from the fundamental core mode to forward propagating cladding modes. The resonant wavelength $\lambda_{\text {res }}$ of an LPG with period $\Lambda$ is determined by the phase matching condition [12]:

$$
\lambda_{\text {res }}=\left(n_{\mathrm{co}}-n_{\mathrm{cl}, \mathrm{m}}\right) \cdot \Lambda,
$$

where $n_{\mathrm{co}}$ and $n_{\mathrm{cl}, \mathrm{m}}$ are the effective indices of the fundamental core mode and the $m$ th cladding mode, respectively. The 


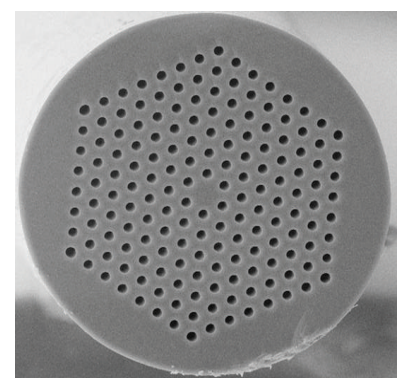

(a)

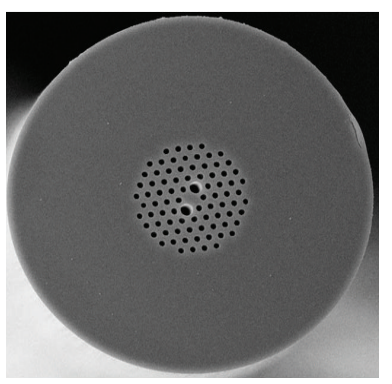

(b)

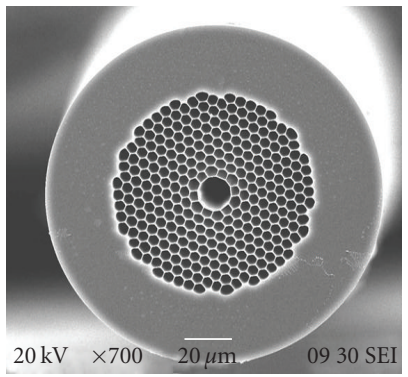

(c)

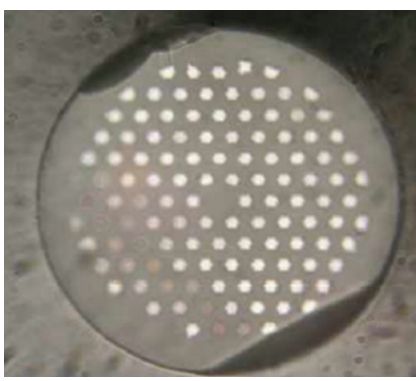

(d)

FIGURE 1: Scanning electron micrographs of different PCF cross-sections. (a) a large-mode-area air-silica endlessly single-mode PCF, (b) an air-silica highly birefringent PCF, (c) an air-silica air-core PBF. These PCFs are from Crystal Fiber A/S [6] (d) an all-solid PBF [5].

cladding modes have higher loss than the core mode, leading to attenuation bands in the transmission spectrum.

Conventional single-mode fibers (SMFs) have Ge-doped cores that are photosensitive and LPGs in such fibers can be formed by periodical perturbing the core refractive index by UV illumination through an amplitude mask or a point-by-point writing technique. LPGs in SMFs have been investigated extensively for applications such as wavelength filters, gain flatteners for EDFAs, and sensors.

PCFs are typically made of a single material (silica) which has no photosensitivity; inscription of PCFs by direct UV irradiation is then difficult. However, LPGs in PCFs have been made by use of nonphotochemical inscription techniques such as $\mathrm{CO}_{2}$ laser irradiation [7], electrical arc discharge [8], external mechanical pressure [13], and femtosecond laser radiation [14].

2.1. LPGs in IG-PCFs. Figure 2(b) shows the side view of an LPG created on a large-mode-area endlessly single-mode PCF (LMA-10) with its cross-section shown in Figure 2(a). The LPG was fabricated by use of a pulsed $\mathrm{CO}_{2}$ laser with a setup described in [7]. Observable notches or grooves were created along the surface of the PCF, indicating considerable collapsing of the air-holes in the cladding region. The LPG has 40 periodic notches along the fiber, and the period is $410 \mu \mathrm{m}$. The transmission spectrum of the LPG, as shown in Figure 3, has double resonant peaks from 1200 to $1700 \mathrm{~nm}$, corresponding to coupling of two different cladding modes. The resonant wavelengths of LPGs in such PCFs were found to decrease with the grating period, which is contrary to that in a conventional SMF.

LPGs with a similar transmission spectrum as shown in Figure 3 can be made with no visible physical deformation (notches) on the surface of the PCFs. The creation of such LPGs requires lower $\mathrm{CO}_{2}$ laser energy density, and in such a LPG, the perturbation of the fiber geometry is negligible and the index perturbation of the glass material is believed to play a significant role in the formation of the LPGs.

The responses of the resonant wavelength to tensile strain are very different for LPGs with and without visible notches [7]. Figure 4 shows the strain and temperature responses of the LPG $\left(\mathrm{LPG}_{1}\right)$ shown in Figure 2 and a similar LPG $\left(\mathrm{LPG}_{2}\right)$ with the same grating length and pitch but no

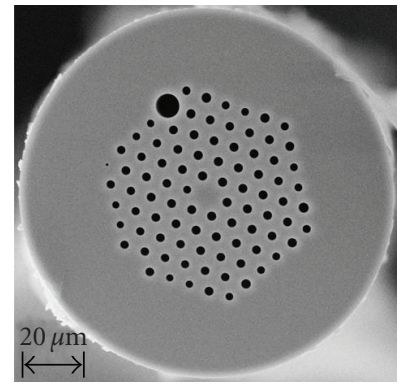

(a)

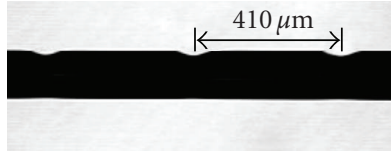

(b)
FIGURE 2: (a) Scanning electron micrograph of the cross-section of LMA-10 PCF, (b) photograph of the LPG with periodic notches [7].

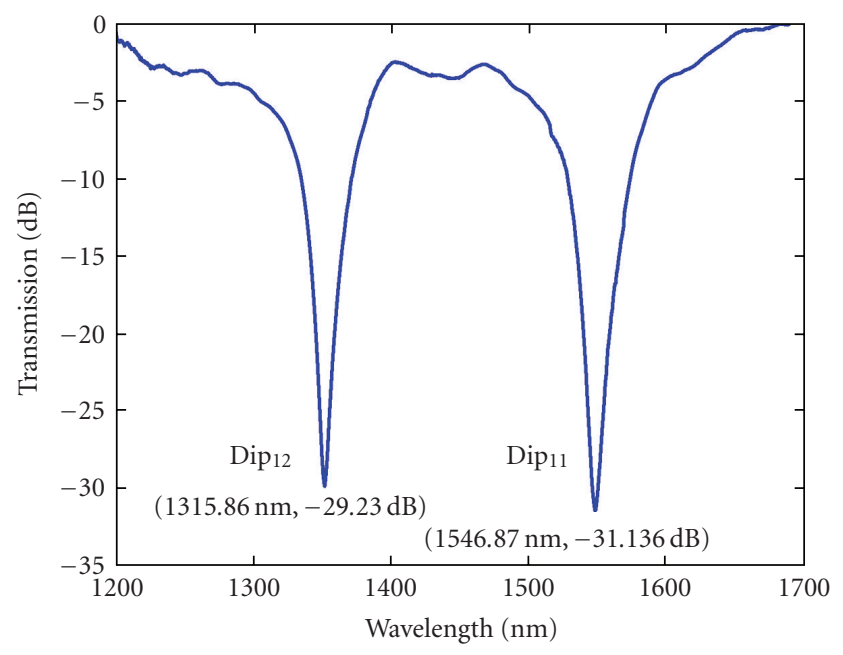

Figure 3: Transmission spectrum of a 40-notch LPG.

observable notches on the fiber surface. $\mathrm{LPG}_{2}$ was fabricated by use of the same $\mathrm{CO}_{2}$ laser setup as for $L P G_{1}$ but a lower dosage of $\mathrm{CO}_{2}$ irradiation. The strain sensitivity of the LPG with notches $(-7.6 \mathrm{pm} / \mu \varepsilon)$ is about 25 times higher than the LPG without physical deformation $(-0.31 \mathrm{pm} / \mu \varepsilon)$. The temperature sensitivities of the two LPGs are approximately the same. The asymmetrical structure caused by the periodic 


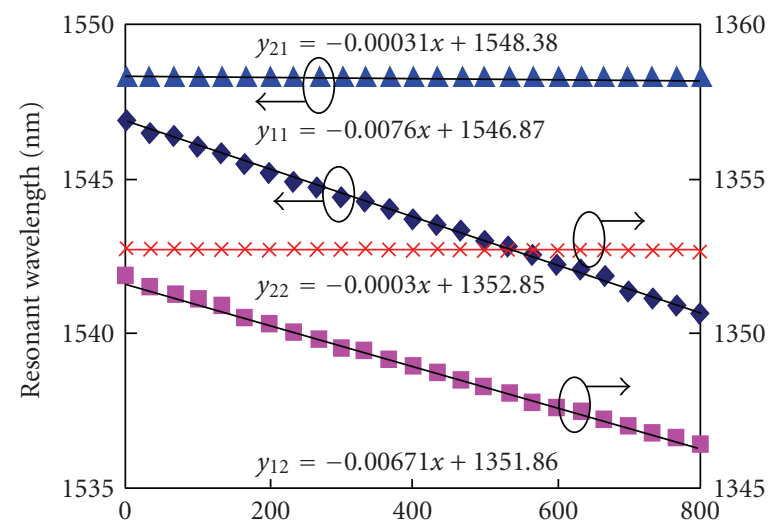

(a)

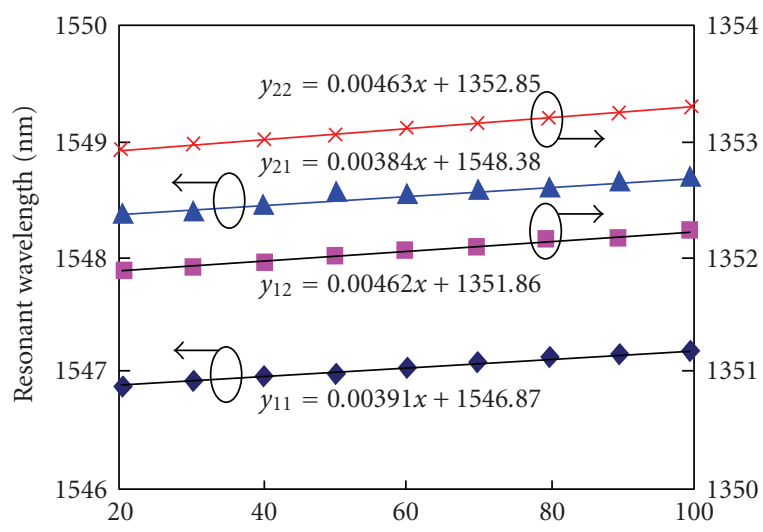

(b)

FIGURE 4: Variation of resonant wavelength of a LPG with notches $\left(\mathrm{LPG}_{1}\right)$ and a similar LPG without notches $\left(\mathrm{LPG}_{2}\right)$ with (a) tensile strain,

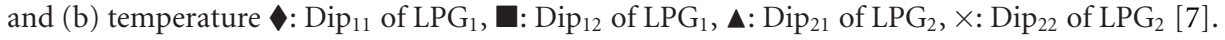

notches introduces microbend when the LPG is axially stretched, which effectively enhanced the refractive index change of the LPGs with notches. The $\mathrm{CO}_{2}$-laser-notched LPGs on PCFs also demonstrated very strong polarization dependent loss (PDL) and can be used as in-fiber polarizers with good temperature stability [15]. The large PDL may be attributed to the side exposure of $\mathrm{CO}_{2}$ laser, which introduces asymmetrical index profile in the fiber cross-section.

Table 1 lists the strain and temperature sensitivities of some PCF-based LPGs fabricated by $\mathrm{CO}_{2}$ laser and electric arc discharge techniques. The sensitivities of SMF-based LPGs are also listed in the table for comparison. The exceptional low temperature dependence of the LPG is regarded as the results of single material used for PCF. As both the cladding and core modes are mostly distributed in the silica, they show similar responses to the temperature change. The very small temperature sensitivity makes them useful for strain measurement with negligible temperature cross-sensitivity.

2.2. LPGs in PBFs. LPGs were also fabricated in hollowcore (HC) PBFs [19]. Figure 5(c) shows the side view of an LPG made on an HC PBF (HC-1550-02) in which periodic notches are created along the fiber. The cross-section, as shown in Figure 5(b), of the PBF at the notched region is asymmetric due to the collapse of air holes and the ablation of glass on one side of the fiber. The outer rings of air holes in the cladding, facing to the $\mathrm{CO}_{2}$ laser irradiation, were largely deformed; however, little or no deformation was observed in the innermost ring of air-holes and in the hollow core.

Figure 5(d) shows the measured transmitted spectrum of a 40 -period LPG. The $3 \mathrm{~dB}$ bandwidth is $\sim 5.6 \mathrm{~nm}$, which is much narrower than that of the LPGs with the same number of grating periods in conventional SMFs and in IG-PCFs $[7,16]$. The insertion loss of the LPG is very low $(<0.3 \mathrm{~dB})$, because most light is guided in the hollow-core where no deformation was observed.

Both refractive index perturbation of the glass material and the changes in air-hole sizes, shapes, and locations may contribute to the formation of the LPG. However, as most light power of the fundamental mode $(>95 \%)$ is in the air region, the effect of refractive index variation of the glass material on the mode index is expected to be smaller than that for conventional fibers and solid core IG-PCFs. On the other hand, as shown in Figure 5(b), the deformation of air-holes in the cladding is obvious, which changes the airfilling fraction and the waveguide structure and perturbs the mode fields and effective indexes of the core, surface, and cladding modes. There could also be weak deformation of the hollow-core, although it is not observable in our experiments. We believe that the periodic perturbation of the waveguide geometry is the dominant factor that causes resonant mode coupling, although the induced refractive index variation may also contribute a little.

A number of LPGs with different pitches and the same number of grating periods were written in the PBG fiber and the measured resonant wavelength as functions of the grating pitch is shown in Figure 5(e), the resonant wavelength decreases with the increase in grating pitch, which is opposite to the LPGs in the conventional SMFs $[16,17]$. For each of the LPGs, two attenuation pits, as shown in Figure 5(e), were observed within 1500 to $1680 \mathrm{~nm}$, indicating that the fundamental mode is coupled to two different higher-order (surface-like) modes.

The responses of the LPG in the air-core PBF to strain, temperature, bend, and external refractive index are measured, and the results are shown in Figure 6. The wavelength sensitivity to strain $(-0.830 \mathrm{pm} / \mu \varepsilon)$ is similar to that of LPGs in IG-PCFs or conventional SMFs (Table 1), and the temperature sensitivity $\left(\sim 2.9 \mathrm{pm} /{ }^{\circ} \mathrm{C}\right)$ is one to two orders of magnitude less than those of the LPGs in the conventional SMFs. In addition, the wavelength sensitivity to bend is three to four orders of magnitude less than those of the LPGs in the conventional SMFs, and it is insensitive to the external refractive index changes. The LPG in HC-PBF may be used as a strain sensor without cross-sensitivity to temperature, curvature, and external refractive index. 
TABLE 1: Strain and temperature sensitivities of LPGs in IG-PCFs by different methods.

\begin{tabular}{|c|c|c|c|c|c|c|}
\hline Inscriptionmethod & Period $(\boldsymbol{\mu} \mathbf{m})$ & Length $(\mathbf{m m})$ & Wavelength $(\mathbf{n m})$ & $\mathrm{d} \lambda / \mathrm{d} \varepsilon(\mathrm{pm} / \mu \varepsilon)$ & $\mathrm{d} \lambda / \mathrm{dT}\left(\mathrm{pm} /{ }^{\circ} \mathrm{C}\right)$ & Reference \\
\hline $\mathrm{CO}_{2}$ laser (with grooves) & 410 & 16.4 & 1546.87 & -7.6 & 3.91 & {$[5]$} \\
\hline $\mathrm{CO}_{2}$ laser (without grooves) & 410 & 16.4 & 1548.38 & -0.31 & 3.84 & {$[5]$} \\
\hline Arc discharge & 400 & 37.6 & 1668 & -2.5 & 3.4 & {$[6]$} \\
\hline Arc discharge & 1000 & 19.0 & 1403 & -2.08 & 2.2 & {$[6]$} \\
\hline $\mathrm{CO}_{2}$ laser* & & & 1526.5 & -0.45 & 58 & {$[16]$} \\
\hline UV exposure* & 320 & & 1573.1 & 7.31 & 46 & {$[17]$} \\
\hline Arc discharge* & 540 & & 1580 & $\sim 1.5$ & $66-73$ & {$[18]$} \\
\hline
\end{tabular}

$*$ Conventional single-mode fiber-based LPG.

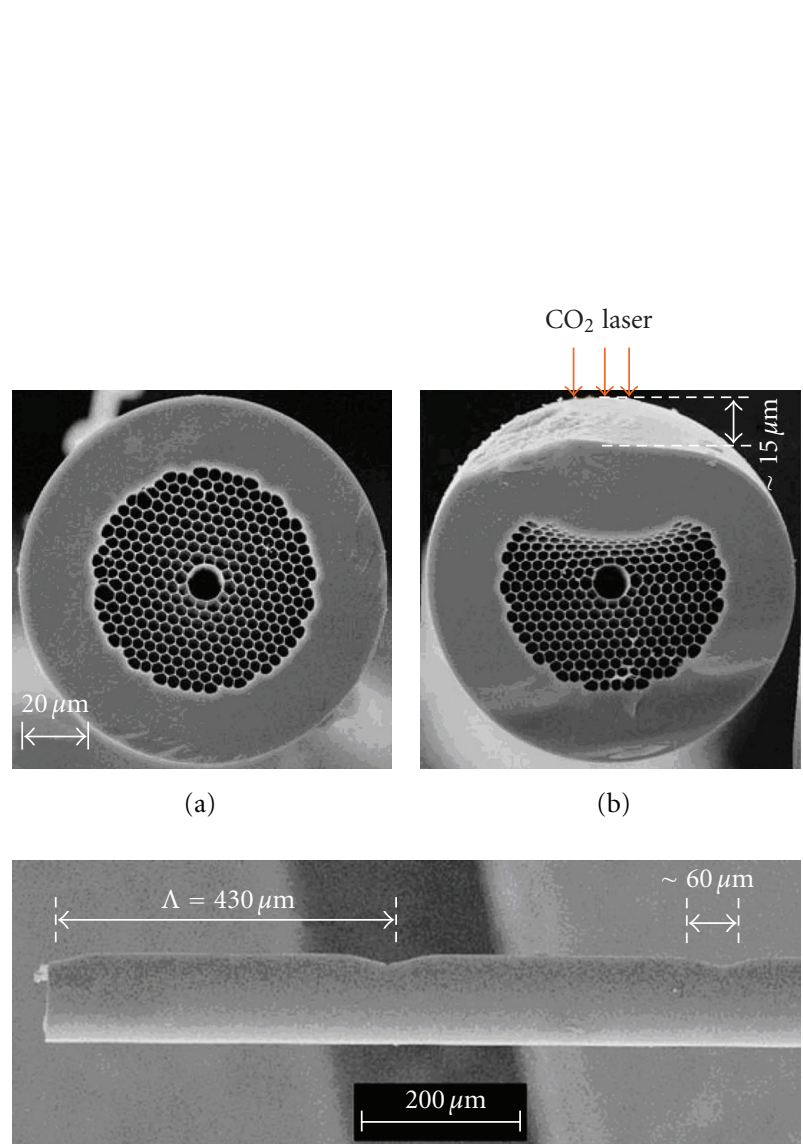

(c)

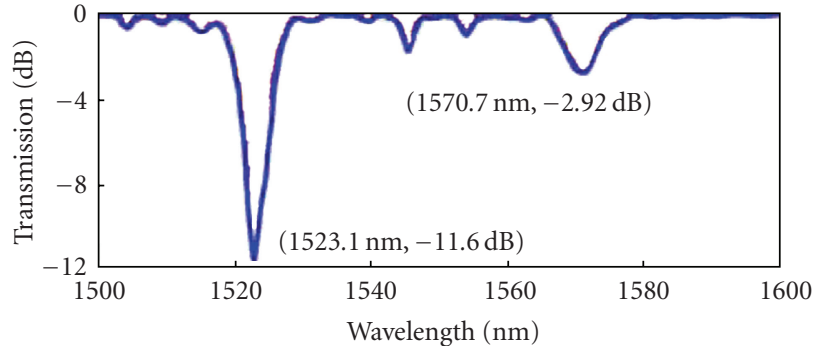

(d)

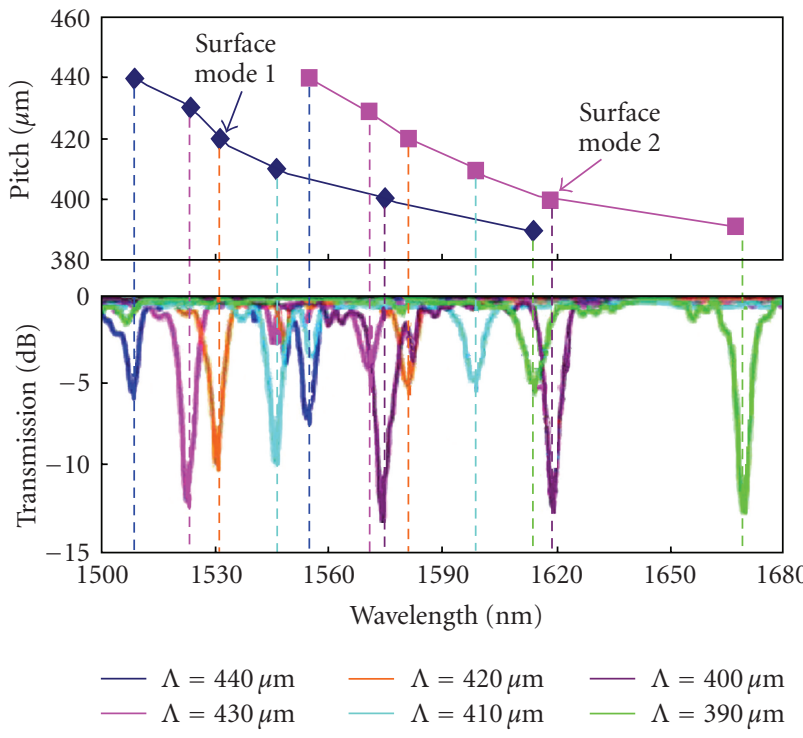

(e)

Figure 5: Scanning electron micrographs of PBF cross-sections (a) before and (b) after $\mathrm{CO}_{2}$ laser irradiation. (c) Periodic notches created on the surface of the PBF. (d) The transmitted spectrum of LPG with 40 periods and a grating pitch of $430 \mu \mathrm{m}$. (e) Variation of LPG resonant wavelengths with grating pitch (upper panel) and the corresponding transmission spectrums (lower panel) [19].

LPGs were also inscribed in all-solid PBG PCF (Figure 1(c)) by the UV exposure method [5]. The allsolid PBG PCF has a solid central silica core region and a microstructured cladding region where higher index Gedoped silica rods are embedded into silica background. The resonant wavelength of the LPG experiences a red shift when the temperature is increased, and the temperature sensitivity is measured to be $19.1 \mathrm{pm} /{ }^{\circ} \mathrm{C}$. This value is higher than those of the PCFs made from a single material.

\section{Modal Interferometric Sensors}

Interferometric sensors have been explored in sensing largely because of their high sensitivities to a broad range of parameters. The modal interferometers in which the interferometric phase difference is accumulated by considering the difference in the effective refractive indices of different fiber modes have been widely investigated. They are attractive for several reasons, including small size, flexibility, as well as the reduced thermal sensitivity in view of the small difference of the 


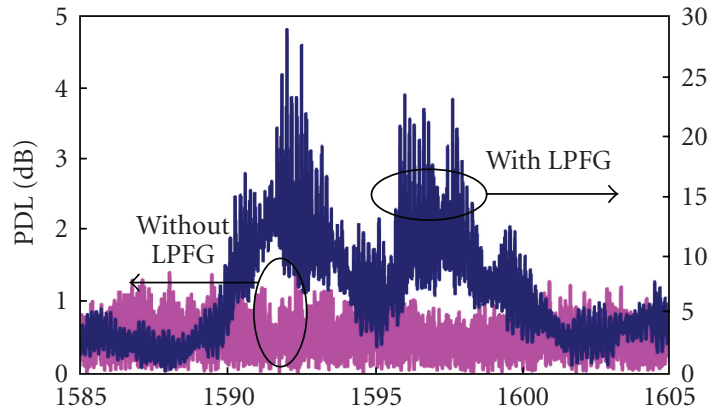

(a)

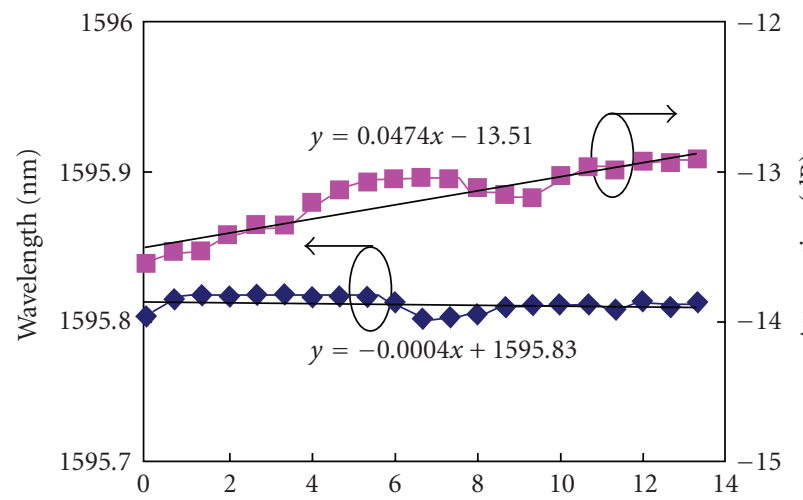

(c)

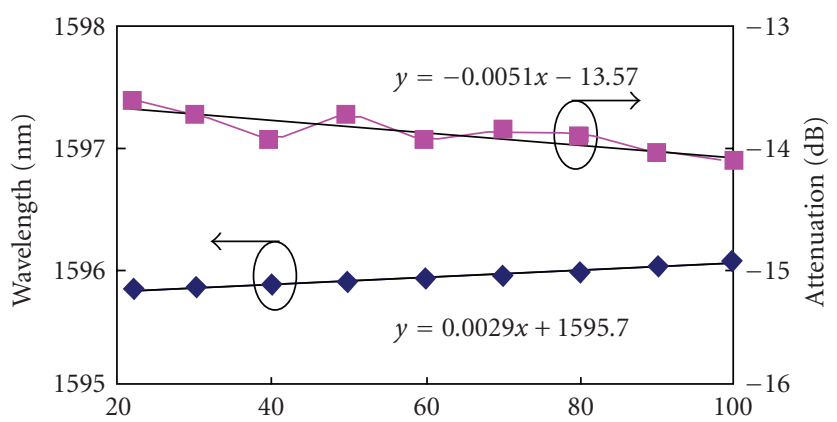

(b)

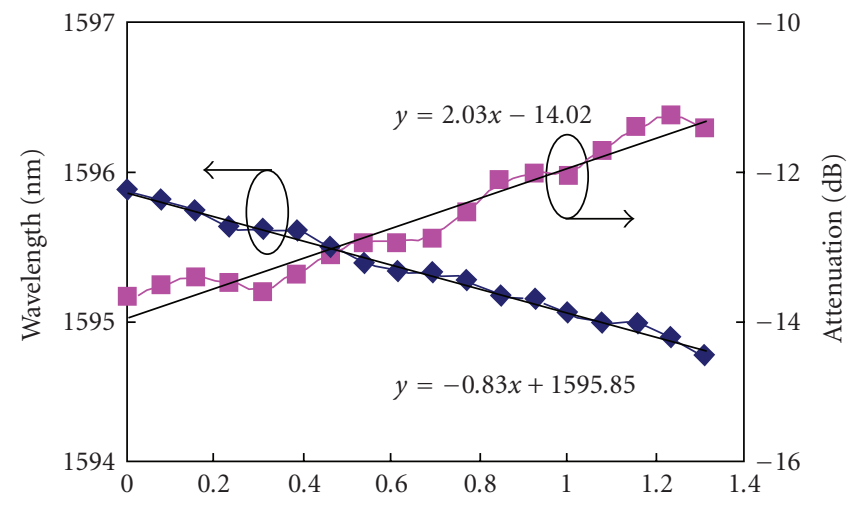

(d)

FIGURE 6: (a) PDL of PBF with and without an LPG. Measured resonant wavelength and peak transmitted attenuation of the LPG as functions of temperature (b), curvature (c), and tensile strain (d) [19].

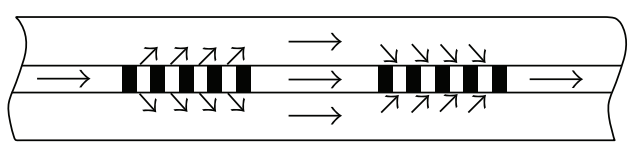

FIgURE 7: A typical MZI configuration.

thermo-optic coefficients of different modes as they travel the same length of optical fibers. In this section, modal interferometers based on different types of modes in IGPCFs will be described and their application to the strain and temperature measurement will be discussed.

3.1. Core/Cladding Modal Interferometer. A core/cladding modal interferometer utilizes the interference between a core mode and a copropagating cladding mode, which is also referred as in-fiber Mach-Zehnder interferometer (MZI). It has been widely used for wavelength filtering and optical sensing $[20,21]$. In the typical MZI configuration as shown in Figure 7, two cascaded LPGs are commonly used with the first LPG couples part of the core mode power into a forward-propagating cladding mode and the second LPG combines the two modes, resulting in sharp interference fringes. The two LPGs serve as beam-splitter/combiner and the core and the cladding modes travel through two independent paths along the same fiber.

A variety of PCF-based MZIs have been demonstrated with modifications on the basic MZI configuration. The first PCF-based MZI has two nearly identical LPGs which are formed by applying periodic mechanical stress to the PCF [22]. Alternatively, all-PCF MZIs can also be formed by offset-splicing combined with partial collapsing of air-holes [23], or by using a single LPG in combination with a short section of PCF where air-holes are fully collapsed [24]. With the advancement of LPG writing technique by using pulsed $\mathrm{CO}_{2}$ laser, the resonant wavelength and attenuation of the LPG can be precisely controlled, which allows compact infiber MZI with low insertion loss to be developed on PCF [25].

Figure 8 shows the wavelength domain interference fringes of an in-fiber MZI formed by a pair of LPGs fabricated directly on PCF by use of a pulsed $\mathrm{CO}_{2}$ laser. The measured strain and temperature responses of the interference peak wavelength at $1646 \mathrm{~nm}$ are shown in Figure 9 . The strain sensitivity is $-2.6 \mathrm{pm} / \mu \mathcal{\varepsilon}$, which agrees with the results reported in elsewhere $(-2.28 \mathrm{pm} / \mu \varepsilon$ at $1560 \mathrm{~nm}$ in [23], and $-2.80 \mathrm{pm} / \mu \varepsilon$ at $1550 \mathrm{~nm}$ in [24]) and is about 5 times higher than that for an MZI fabricated on a standard SMF $(+0.445 \mathrm{pm} / \mu \varepsilon$, Figure $9(\mathrm{~b}))$ with the same $\mathrm{CO}_{2}$ laser technique and two times higher than that of a typical fiber Bragg grating (FBG) sensor. The temperature sensitivity of the MZI is measured to be $42.4 \mathrm{pm} /{ }^{\circ} \mathrm{C} \cdot \mathrm{m}$, which is almost 30 times lower than that of the MZI in the SMF $\left(1215.56 \mathrm{pm} /{ }^{\circ} \mathrm{C} \cdot \mathrm{m}\right.$, Figure 9(a)) [25].The very small temperature sensitivity may be attributed to the single material property of the PCF, which results in similar 


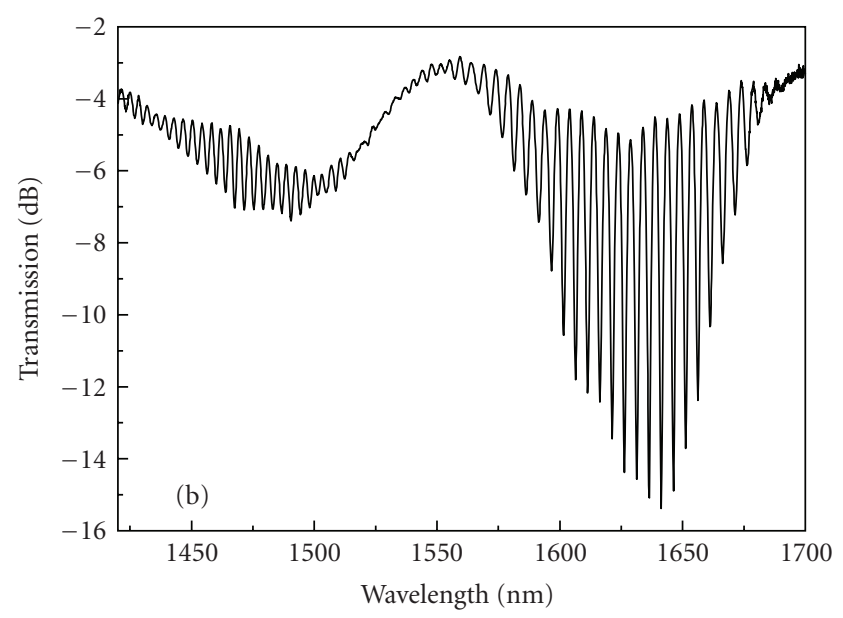

Figure 8: The transmission spectrum of a PCF-MZI with a cavity length of $190 \mathrm{~mm}$ [25].

response of the core and cladding modes to temperature. The MZI on PCF may be potentially used as a temperatureinsensitive strain sensor and as stable multichannel filter with low temperature dependence.

3.2. Two-Mode PCF Interferometer. It is possible to make infiber devices based in the interference between the two core modes. This however requires the use of a two-mode fiber, that is, a fiber that supports only two core modes, to avoid interference with other core modes. Conventional step-index two-mode optical fibers have been investigated for various device applications such as wavelength filters, frequency shifters, switches, interferometric strain, and temperature measurement. However, the wavelength range of the twomode operation for conventional fibers is typically less than $150 \mathrm{~nm}$ [26], which limit the potential applications of the two-mode devices.

It has been shown that an air-silica IG-PCF with a crosssection similar to Figure 1(b) can be designed to operate as a two-mode fiber over the entire low-loss transmission window of the silica glass [27]. This would allow the creation of extremely broadband two-mode fiber devices and sensors.

We investigated theoretically and experimentally the modal properties of the commercial high birefringent (HiBi) PCF as shown in Figure 1(b) (PM-1550, Crystal Fiber A/S [6]) and found it operating as a two-mode fiber over wavelength range from below 543 to $1400 \mathrm{~nm}$, although it guides only the fundamental mode at $1550 \mathrm{~nm}$. Figure 10 shows the transverse mode field patterns of the four nondegenerate approximately linearly polarized (LP) modes at $1330 \mathrm{~nm}$, which were calculated by using a full-vector finite element method (FEM). By analogy to the elliptical core fiber, these four modes are labeled as $\operatorname{LP}_{01}{ }^{x}, \operatorname{LP}_{01} y, \operatorname{LP}_{11}$ (even), and $\mathrm{LP}_{11}{ }^{y}$ (even) modes. The superscripts $x$ and $y$ correspond to the $x$ - and $y$-polarizations, respectively. It is interesting to notice that this Hi-Bi PCF is capable to suppress the $\mathrm{LP}_{11}$ (odd) modes. The wide two-mode wavelength range and the stable lobe orientation make it possible to built stable two-mode devices and interferometric sensors with long length of fibers.

A two-mode fiber sensor uses a differential interferometric scheme where the interference between the $\mathrm{LP}_{01}$ and the $\mathrm{LP}_{11}$ (even) modes of the fiber leads to a varying twolobe pattern in the output. When the $\mathrm{LP}_{01}$ and $\mathrm{LP}_{11}$ (even) modes with the same polarization state are excited equally in the Hi-Bi PCF, the output radiation pattern will be a superposition of the contribution from the two modes and will be a function of the relative phase difference $\varphi$ between them. For a change in $\varphi$ of $2 \pi$ there will be one complete oscillation of the intensity pattern.

An external disturbance, for example, strain or temperature, applied on the PCF two-mode interferometer leads to a differential phase shift between these two modes, resulting in an oscillation of the two-lobe pattern. A spatial demodulator monitoring one of the two-lobe patterns converts this oscillation into an intensity variation. When only the $x$ - or $y$-polarization of $\mathrm{LP}_{01}$ and $\mathrm{LP}_{11}$ modes was excited at the entrance, a quasisinusoidal intensity waveform is obtained after the spatial demodulator.

3.2.1. Two-Mode PCF Strain Sensor. Figure 11 is a schematic of the experimental setup used to study the strain response of the two-mode PCF interferometer. Light from a laser was coupled into a piece of Hi-Bi PCF (PM-1550, Crystal Fiber A/S) with an alignment system consisting of a pair of lenses, a polarizer, a fiber holder, and a 5-dimensional translation stage. The PCF has a total length of $\sim 1$ meter and is epoxybounded to a fixed stage and a translation stage. The $50 \mathrm{~cm}$ PCF in between the two stages can be axially strained through a computer controlled translation stage. An infrared TV camera with lens removed is placed near the output of the fiber to monitor one of two lobes of the far-field intensity patterns, as indicated in the rectangular region in the right panel of Figure 11. Alternatively, it is possible to use a leadout fiber which is offset from the two-mode PCF to pick up the maximum contrast ratio in the intensity of the two-lobe output.

Experiments were conducted with different semiconductor lasers with wavelengths of $650,780,850,980$, and $1310 \mathrm{~nm}$. Figure 12 shows the measured intensity variation at one of the lobes at $1310 \mathrm{~nm}$ when the PCF was elongated from 0 to $2 \mathrm{~mm}$. The curves from top to bottom correspond, respectively, to polarizer set to $0^{\circ}, 90^{\circ}$, and $45^{\circ}$, in respect to the $x$-axis as shown in the right panel of Figure 11 . At $0^{\circ}$ and $90^{\circ}$, the intensity variation is due to the interference of $\mathrm{LP}_{01}$ and $\mathrm{LP}_{11}$ (even) modes for the $x$ - and $y$-polarizations, respectively. At a launch angle of $45^{\circ}$ respective to the principle axis of PCF, the two sets of interference patterns, corresponding to two orthogonal polarizations, are superimposed, resulting in an amplitude-modulated wave as shown in the lower graph of Figure 12.

The strain sensitivities for orthogonal polarizations, which are defined as the rate of change of the phase difference between the two modes with respect to strain, are shown in Figure 13. The strain sensitivities have linear relationship 


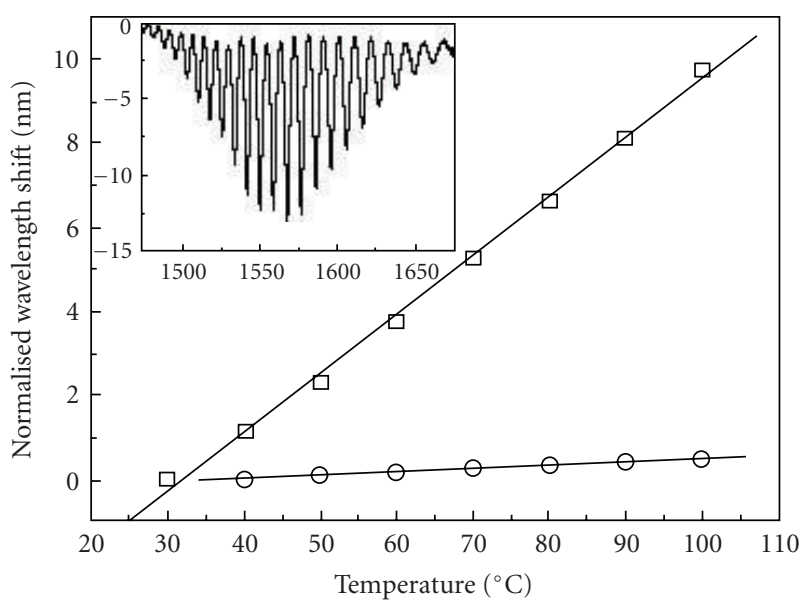

(a)

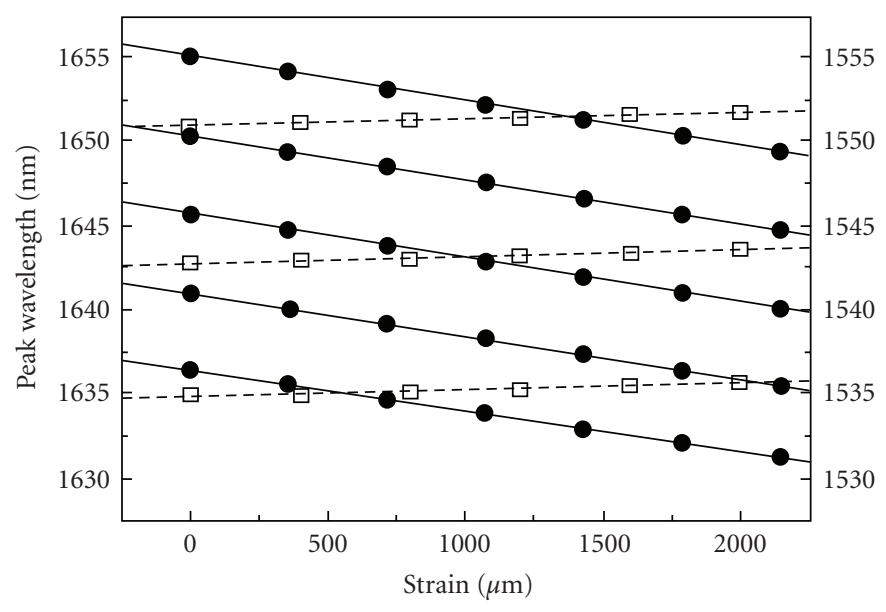

(b)

FIGURE 9: (a) Temperature responses of the PCF-MZI with transmission spectrum in Figure 8 (circular points) and an SMF-MZI with output spectrum shown in the inset (rectangular points). Solid lines: linear fits. (b) Strain responses of the interference peaks as functions of strain (PCF-MZI: left axis, filled circles, SMF-MZI: right axis, open squares). The solid and dash lines are the corresponding linear fits for the PCF-MZI and SMF-MZI [25].

with the optical wavelength, and higher sensitivity is achieved at longer wavelength.

3.2.2. Two-Mode PCF Temperature Sensor. The experimental setup used for studying the temperature response is similar to that shown in Figure 11, except that a section of PCF $(\sim 1.8$ meters $)$ was heated by putting it inside an oven. Measurements were performed for both the $x$ - and $y$ polarizations. Figure 14 shows an example of the output intensity of the two-mode interferometer at $1310 \mathrm{~nm}$ as a function of oven temperature when the input polarization is aligned to the $x$-axis. One complete cycle in the intensity variation corresponds to a $2 \pi$ change in the phase difference between the $\mathrm{LP}_{01}$ and $\mathrm{LP}_{11}$ (even) modes. We noticed that the period of the intensity oscillation is not constant during the temperature range from $20^{\circ} \mathrm{C}$ to $120^{\circ} \mathrm{C}$, and the period is obviously larger at lower temperatures, indicating that the interferometer shows a nonlinear response to the temperature changes. The temperature sensitivity $\eta$, defined as the rate of change of phase difference $\Delta \phi$ between the two modes with respect to temperature $T$ per unit length of sensing fiber, is given by

$$
\eta=\frac{1}{L} \cdot \frac{\Delta \phi}{\Delta T}
$$

where $L$ is the fiber length placed inside the oven. The measured average temperature sensitivities as functions of wavelength within the temperature range from $20^{\circ} \mathrm{C}$ to $120^{\circ} \mathrm{C}$ are shown in Figure 15. The temperature sensitivities of two-mode PCF sensor are slightly different for the $x$ and $y$-polarizations. And the values are in general smaller than those of the conventional elliptical core two-mode fibers [29].

The extremely broad two-mode wavelength range and the unusual wavelength-dependent temperature sensitivity of the two-mode PCF sensor provide a useful means for strain and temperature discrimination. As shown in Figure 13, the strain sensitivities increase linearly with wavelength and are significantly different $(\sim 15 \%)$ for the two orthogonal polarizations. The temperature sensitivities have nonmonotonic dependence on wavelength (Figure 15) and are similar for both polarizations. A temperatureindependent strain measurement can then be realized by operating the interferometer at two wavelengths where the temperature sensitivities are the same, and taking a differential measurement. On the other hand, it is possible to use the two-mode sensor for simultaneous measurement of strain and temperature by operating at two properly chosen optical wavelengths.

\section{Conclusions}

In conclusion, PCF provides a new platform for the development of optical fiber sensors. It allows for LPGs with exceptional low temperature coefficient to be created, while the strain sensitivity can be significantly enhanced by carving visible grooves on the surface of the fiber. The LPG fabricated in the HC-PBF has many advantages compared with those in conventional SMFs and index-guiding PCFs such as insensitivity to temperature, bending, and external refractive index. It is also possible to construct an interferometric strain or temperature sensor by utilizing the interference between the fundamental core mode and cladding mode or a higherorder core mode. The core/cladding mode interferometric sensor based on PCF has higher strain sensitivity and lower temperature sensitivity than its SMF counterpart. The interference between two core modes of PCF can be operated over a broader wavelength range than conventional twomode fibers. The strain sensitivity of two-mode PCF sensor is comparable to that of the conventional two-mode sensors and shows a linear relationship with optical wavelength. The 


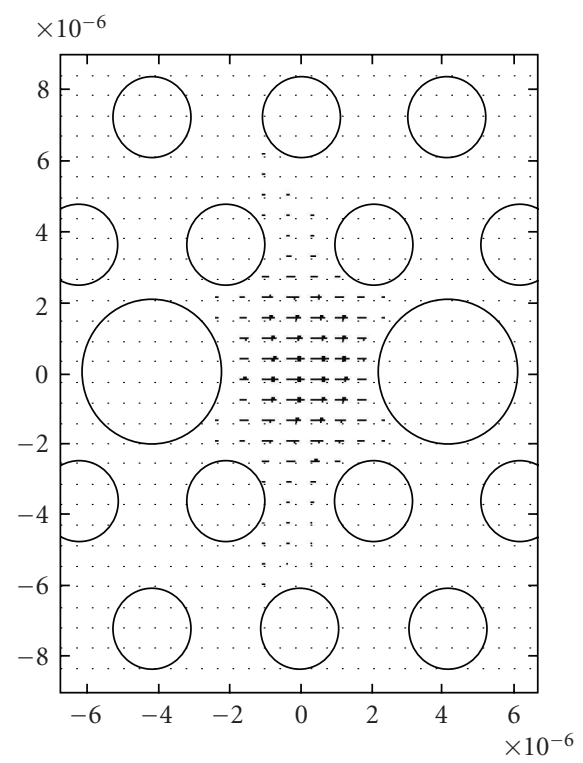

(a)

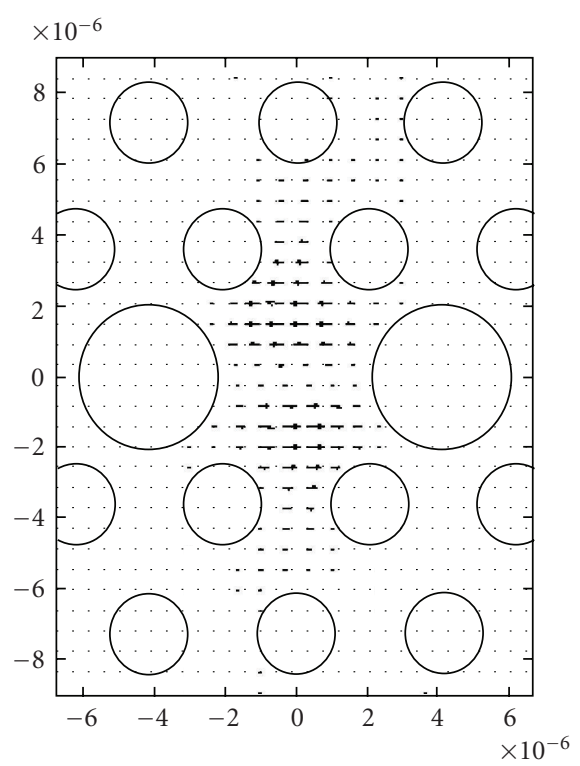

(c)

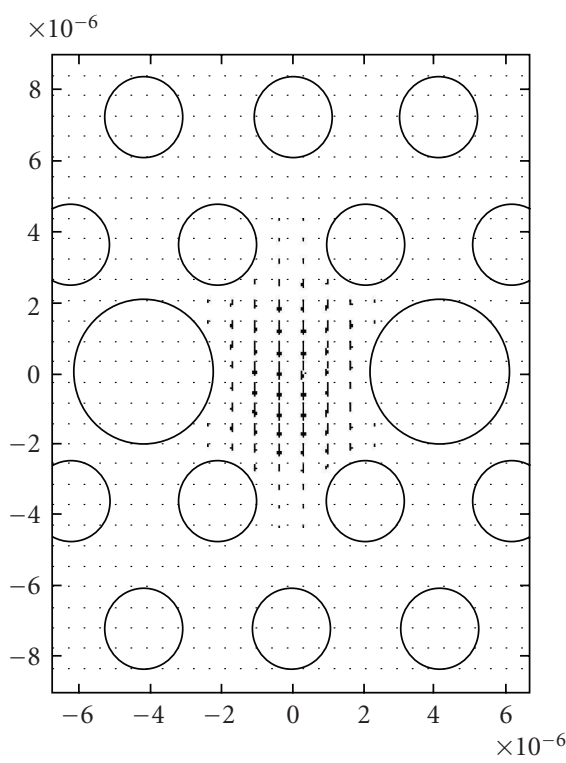

(b)

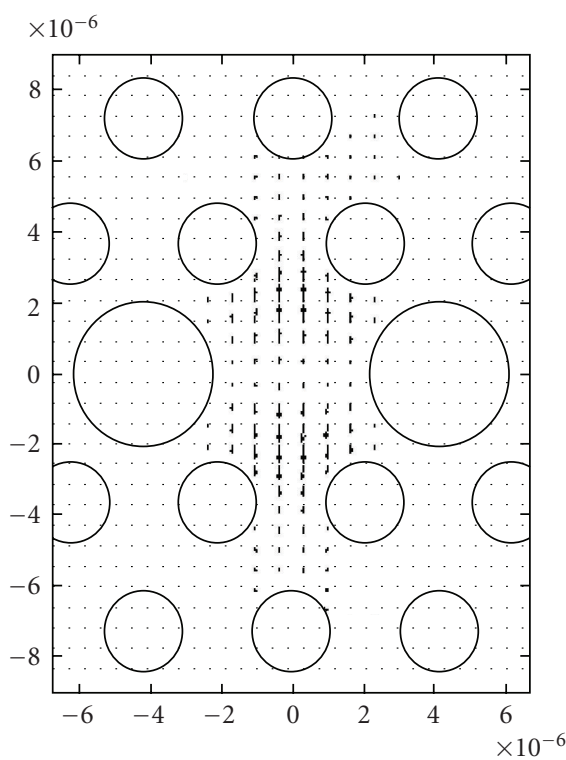

(d)

Figure 10: Transverse electric field distribution of (a) $\mathrm{LP}_{01}{ }^{x}$, (b) $\mathrm{LP}_{11}{ }^{y}$, (c) $\mathrm{LP}_{01}{ }^{x}$ (even), and (d) $\mathrm{LP}_{11}{ }^{y}$ (even) modes for an Hi-Bi PCF operating at $1.3 \mu \mathrm{m}$.

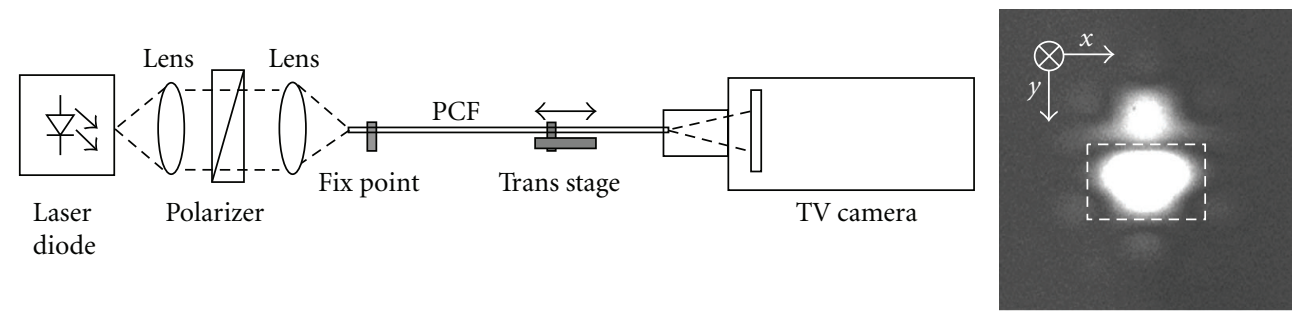

FIgURE 11: Experimental setup for strain measurement and far-field intensity pattern. 


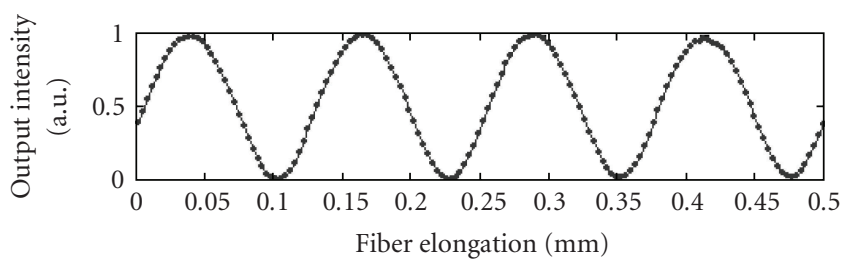

(a)

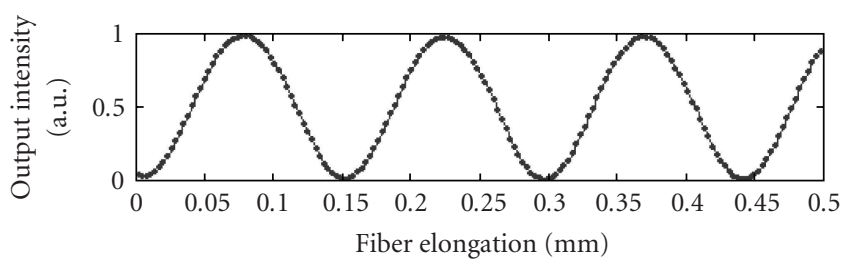

(b)

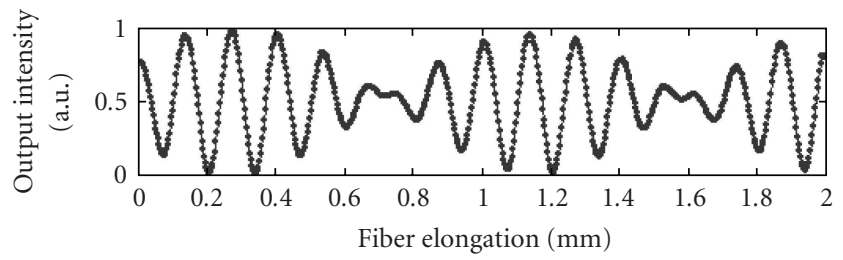

(c)

FIGURE 12: Experimental results for different launch angles of $0^{\circ}$, $90^{\circ}$, and $45^{\circ}$ (from top to bottom) [28].

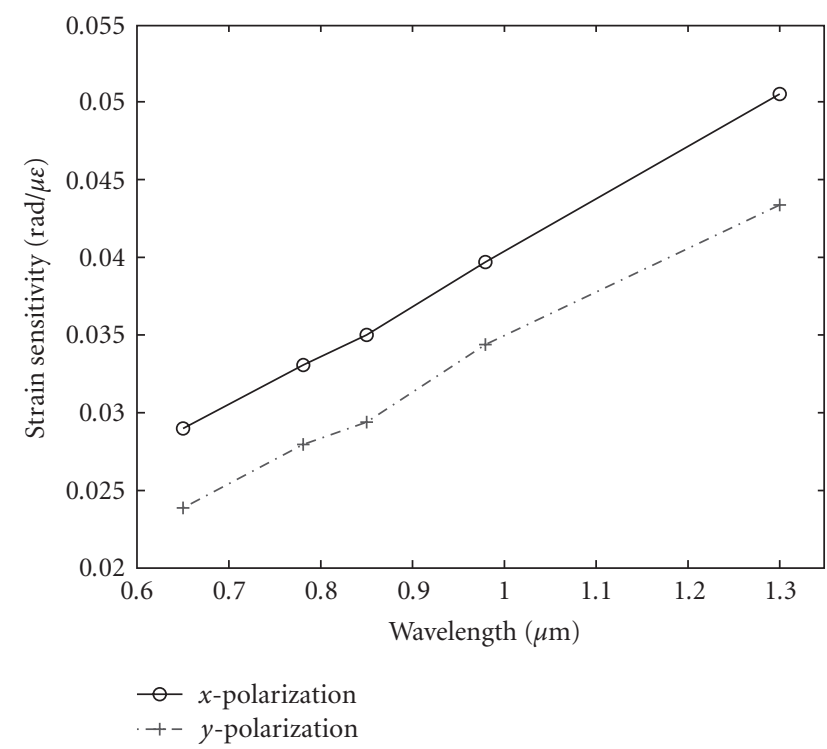

FIgURE 13: Strain sensitivity as function of wavelength.

temperature sensitivity of the two-mode PCF sensor is in general smaller than that of the conventional elliptical core two-mode fiber sensors and has a nonmonotonic relationship with optical wavelength, which may be employed for temperature-independent strain sensing by operating at two wavelengths with similar temperature coefficients.

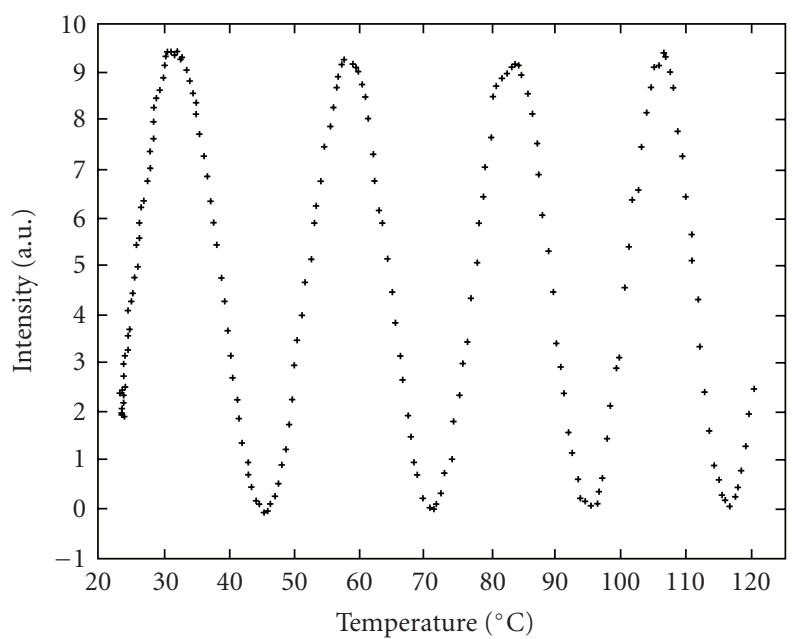

Figure 14: Experimental results showing the periodic intensity variation with temperature. $(\lambda=1310 \mathrm{~nm}, x$-polarization $)$ [30].

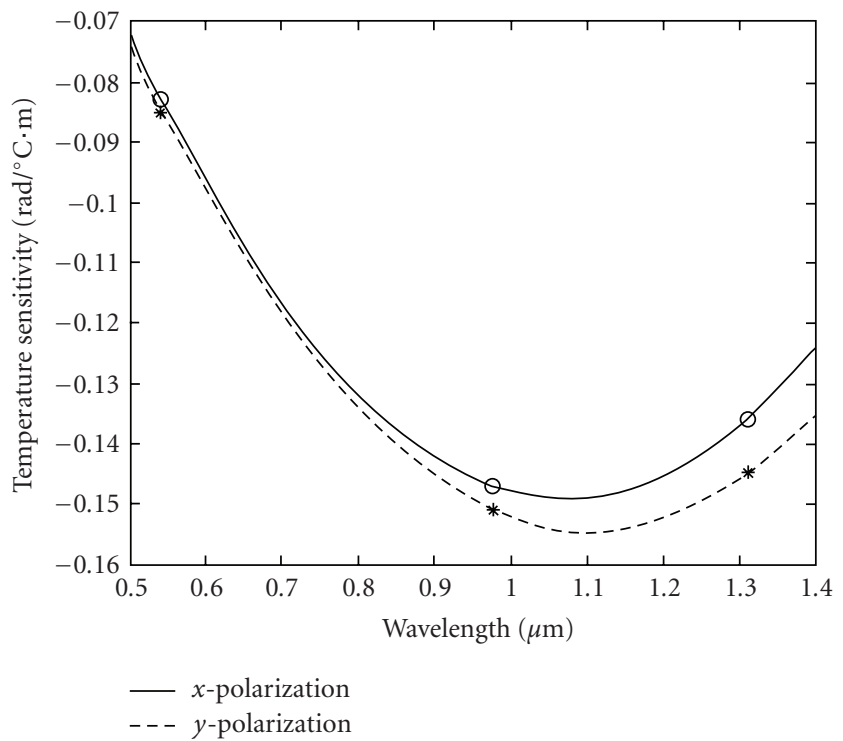

FIGURE 15: Measured temperature sensitivity for $x$-polarization (circle) and $y$-polarization (star) as a function of optical wavelength. Solid and dashed lines are curve fitting results of the measured data [30].

\section{Acknowledgments}

The research work was partially supported by NSF of China through Grant 60629401, and the Hong Kong SAR Government through GRF Grants PolyU 5182/07E.

\section{References}

[1] P. Russell, "Photonic crystal fibers," Science, vol. 229, pp. 258362, 2003.

[2] T. A. Birks, J. C. Knight, and P. St. J. Russell, "Endlessly singlemode photonic crystal fiber," Optics Letters, vol. 22, no. 13, pp. 961-963, 1997.

[3] K. Suzuki, H. Kubota, S. Kawanishi, M. Tanaka, and M. Fujita, 
"Optical properties of a low-loss polarization-maintaining photonic crystal fiber," Optics Express, vol. 9, no. 13, pp. 676680, 2001.

[4] R. F. Cregan, B. J. Mangan, J. C. Knight, et al., "Single-mode photonic band gap guidance of light in air," Science, vol. 285, no. 5433, pp. 1537-1539, 1999.

[5] L. Jin, Z. Wang, Y. Liu, G. Kai, and X. Dong, "Ultravioletinscribed long period gratings in all-solidnphotonic bandgap fibers," Optics Express, vol. 16, no. 25, pp. 21119-21131, 2008.

[6] http://www.nktphotonics.com.

[7] Y.-P. Wang, L. Xiao, D. N. Wang, and W. Jin, "Highly sensitive long-period fiber-grating strain sensor with low temperature sensitivity," Optics Letters, vol. 31, no. 23, pp. 3414-3416, 2006.

[8] H. Dobb, K. Kalli, and D. J. Webb, "Measured sensitivity of arcinduced long-period grating sensors in photonic crystal fibre," Optics Communications, vol. 260, no. 1, pp. 184-191, 2006.

[9] Y. Zhu, Z. He, and H. Du, "Detection of external refractive index change with high sensitivity using long-period gratings in photonic crystal fiber," Sensors and Actuators B, vol. 131, no. 1, pp. 265-269, 2008.

[10] Z. He, Y. Zhu, and H. Du, "Effect of macro-bending on resonant wavelength and intensity of long-period gratings in photonic crystal fiber," Optics Express, vol. 15, no. 4, pp. 18041810, 2007.

[11] Y. L. Hoo, W. Jin, C. Shi, H. L. Ho, D. N. Wang, and S. C. Ruan, "Design and modeling of a photonic crystal fiber gas sensor," Applied Optics, vol. 42, no. 18, pp. 3509-3515, 2003.

[12] A. M. Vengsarkar, P. J. Lemaire, J. B. Judkins, V. Bhatia, T. Erdogan, and J. E. Sipe, "Long-period fiber gratings as bandrejection filters," Journal of Lightwave Technology, vol. 14, no. 1, pp. 58-64, 1996.

[13] J. H. Lim, K. S. Lee, J. C. Kim, and B. H. Lee, "Tunable fiber gratings fabricated in photonic crystal fiber by use of mechanical pressure," Optics Letters, vol. 29, no. 4, pp. 331333, 2004.

[14] A. A. Fotiadi, G. Brambilla, T. Ernst, S. A. Slattery, and D. N. Nikogosyan, "TPA-induced long-period gratings in a photonic crystal fiber: inscription and temperature sensing properties," Journal of the Optical Society of America B, vol. 24, no. 7, pp. 1475-1481, 2007.

[15] Y.-P. Wang, L. Xiao, D. N. Wang, and W. Jin, "In-fiber polarizer based on a long-period fiber grating written on photonic crystal fiber," Optics Letters, vol. 32, no. 9, pp. 1035-1037, 2007.

[16] Y.-J. Rao, Y.-P. Wang, Z.-L. Ran, and T. Zhu, "Novel fiber-optic sensors based on long-period fiber gratings written by highfrequency $\mathrm{CO}_{2}$ laser pulses," Journal of Lightwave Technology, vol. 21, no. 5, pp. 1320-1327, 2003.

[17] V. Bhatia and A. M. Vengsarkar, "Optical fiber long-period grating sensors," Optics Letters, vol. 21, no. 9, pp. 692-694, 1996.

[18] G. Rego, R. Falate, O. Ivanov, and J. L. Santos, "Simultaneous temperature and strain measurements performed by a step-changed arc-induced long-period fiber grating," Applied Optics, vol. 46, no. 9, pp. 1392-1396, 2007.

[19] Y.-P. Wang, W. Jin, J. Ju, et al., "Long period gratings in aircore photonic bandgap fibers," Optics Express, vol. 16, no. 4, pp. 2784-2790, 2008.

[20] X. J. Gu, "Wavelength-division multiplexing isolation fiber filter and light source using cascaded long-period fiber gratings," Optics Letters, vol. 23, no. 7, pp. 509-510, 1998.

[21] K. T. V. Grattan and B. T. Meggitt, Optical Fiber Sensor Technology; Vol. 2: Devices and Technology, Chapman \& Hall,
London, UK, 1998.

[22] J. H. Lim, H. S. Jang, K. S. Lee, J. C. Kim, and B. H. Lee, "Mach-Zehnder interferometer formed in a photonic crystal fiber based on a pair of long-period fiber gratings," Optics Letters, vol. 29, no. 4, pp. 346-348, 2004.

[23] H. Y. Choi, M. J. Kim, and B. H. Lee, "All-fiber Mach-Zehnder type interferometers formed in photonic crystal fiber," Optics Express, vol. 15, no. 9, pp. 5711-5720, 2007.

[24] H. Y. Choi, K. S. Park, and B. H. Lee, "Photonic crystal fiber interferometer composed of a long period fiber grating and one point collapsing of air holes," Optics Letters, vol. 33, no. 8, pp. 812-814, 2008.

[25] J. Ju, W. Jin, and H. L. Ho, "Compact in-fiber interferometer formed by long-period gratings in photonic crystal fiber," IEEE Photonics Technology Letters, vol. 20, no. 23, pp. 18991901, 2008.

[26] B. Y. Kim, J. N. Blake, S. Y. Huang, and H. J. Shaw, "Use of highly elliptical core fibers for two-mode fiber devices," Optics Letters, vol. 12, pp. 729-731, 1987.

[27] W. Jin, Z. Wang, and J. Ju, "Two-mode photonic crystal fibers," Optics Express, vol. 13, no. 6, pp. 2082-2088, 2005.

[28] J. Ju, W. Jin, and M. S. Demokan, "Two-mode operation in highly birefringent photonic crystal fiber," IEEE Photonics Technology Letters, vol. 16, no. 11, pp. 2472-2474, 2004.

[29] S.-Y. Huang, J. N. Blake, and B. Y. Kim, "Perturbation effects on mode propagation in highly elliptical core two-mode fibers," Journal of Lightwave Technology, vol. 8, no. 1, pp. 2333, 1990.

[30] J. Ju, Z. Wang, W. Jin, and M. S. Demokan, "Temperature sensitivity of a two-mode photonic crystal fiber interferometric sensor," IEEE Photonics Technology Letters, vol. 18, no. 20, pp. 2168-2170, 2006. 

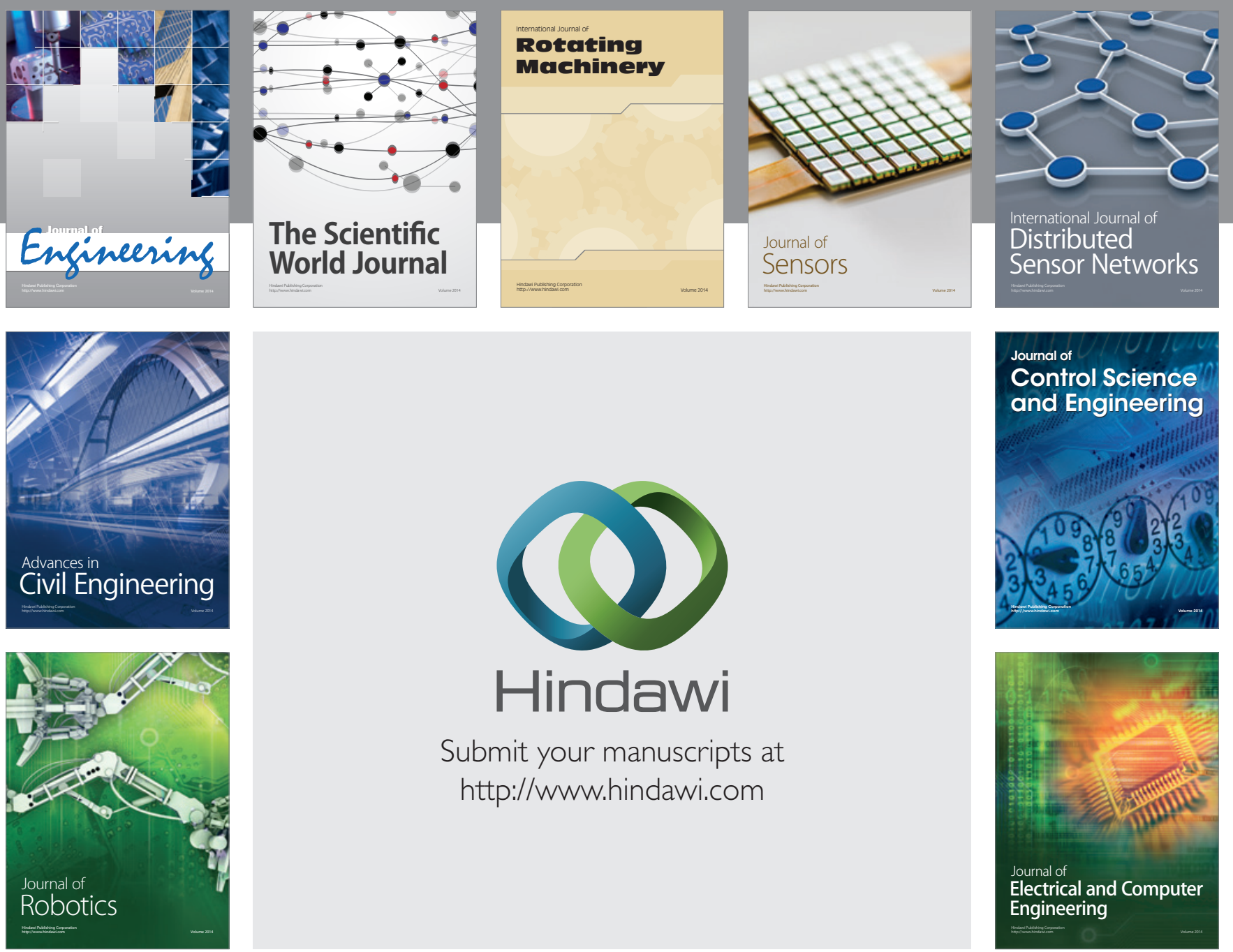

Submit your manuscripts at

http://www.hindawi.com
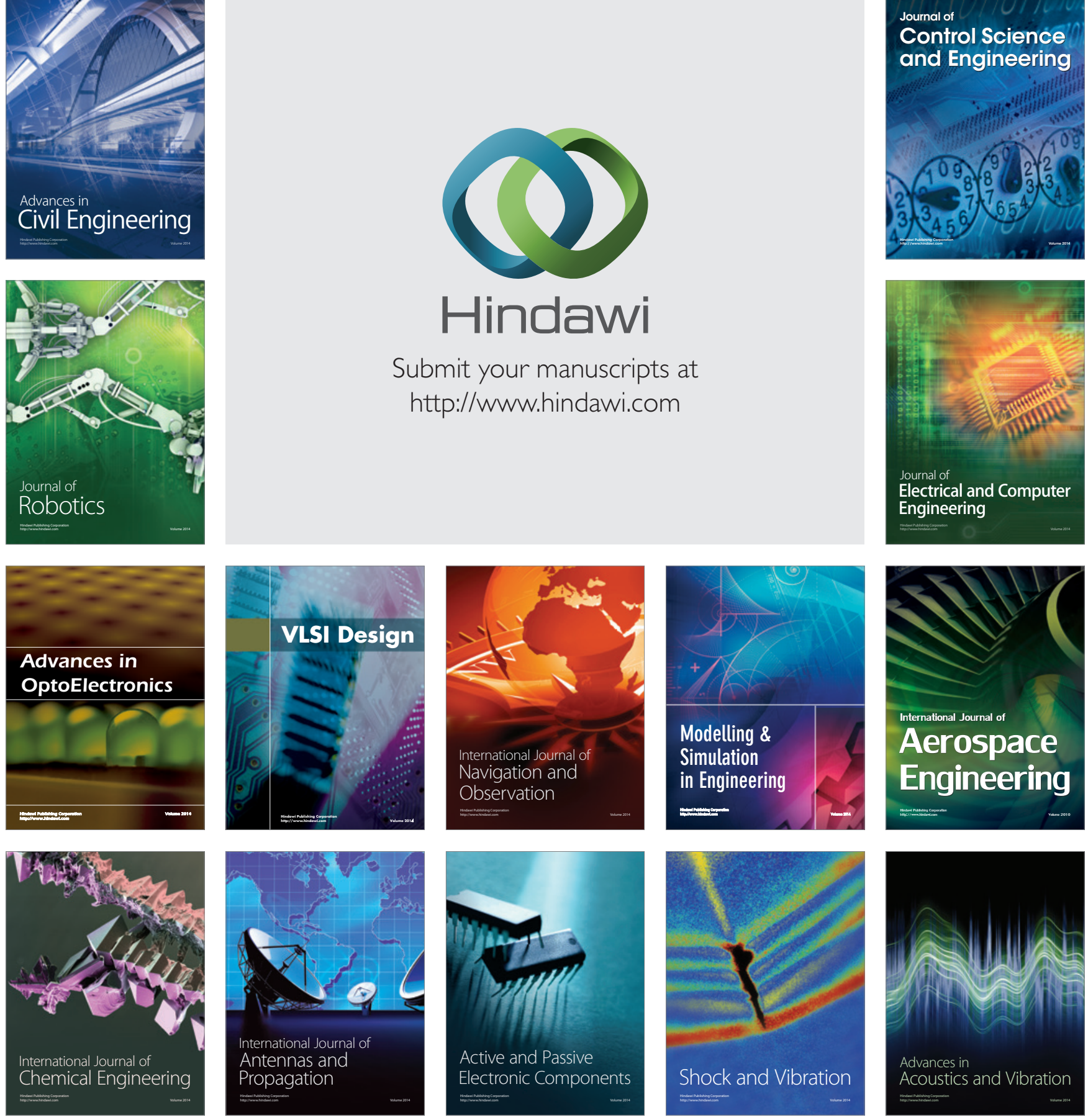